\title{
EDUCACIÓN LATINOAMERICANA: RETOS DE CONSTRUCCIÓN SOCIO-ESPACIAL
}

\author{
Andrés Machuca Téllez \\ Magister en Educación Universidad Libre \\ Corporación Universitaria Nacional de Educación Superior (CUN) \\ andresmachucatellez@gmail.com
}

\section{Cómo citar este artículo:}

Machuca, A. (2014) Educación Latinoamericana: retos de construcción socio-espacial. Espiral, Revista de Docencia e Investigación, 4(2), 27 - 40.

\section{Resumen}

Dos son los elementos que deben ser reflexionados desde la educación en su deber de atender a su responsabilidad social; su relación con las lógicas imperantes de mercado y el reconocimiento del "otro" dentro de las relaciones socio-espaciales que configuran el acto educativo. A continuación se presenta un artículo reflexivo (Palou, 2001) que emerge del abordaje puntual de los planteamientos de Torres (2009), Laval (2004) y Castro (2000) en donde se propone un dialogar alrededor del impacto de la relación del conocimiento social y la educación en búsqueda de construir una conciencia histórica como elemento para el rescatar el Ser, a partir del reconocimiento de realidades socio-espaciales, para lo cual en un primer momento se desarrolla un balance histórico de la relación educación y las realidades socio-espaciales; esto con el fin de mostrar la ligadura entre ambos y su repercusión en los movimientos sociales. En un segundo momento se pondrá en evidencia el problema de los procesos de colonización en el mundo y particularmente en la región comprendida desde el Rio Bravo en México hasta el Cabo de Hornos en Chile, Latinoamérica. Por último, se señalaran los impactos de las políticas educativas, resultado de las lógicas de conocimiento social desarrolladas desde lugares externos a la realidad Latinoamericana y su impacto en la comprensión de la educación. Al final se presentan algunas reflexiones puntuales a manera de conclusión que resaltan la importancia de reconstruir la Universidad Latinoamericana desde el reconocimiento de las realidades socio-espaciales.

Palabras clave: Educación, Humanidades, Socio-espacialidad, Reconocimiento del Otro

\footnotetext{
Abstract

There are two elements that must be reflected upon from education in their duty of caring about its social responsibility; its relationship to prevailing market logic and recognition of the "other" within the socio-spatial
}

relationships that shape the educational act. Then, a thoughtful article (Palou, 2001) that emerges from approaching Torres (2009), Laval (2004) and Castro's (2000) statements where a dialogue about the impact of the relationship of the social knowledge and education seeking to build a historical consciousness as an element to rescue the Being is proposed, from the recognition of socio-spatial realities, for which at a first moment a historical balance of education and socio-spatial realities relationship is developed; this in order to show the bond between them and their impact on social movements. In a second moment, the problem of colonization processes in the world and particularly in the region from the Rio Bravo in Mexico to Cape Horn in Chile, Latin America is placed in evidence. Finally, the impacts of educational policies were highlighted, result of the logics of social knowledge developed from external sites to the Latin American reality and its impact on the understanding of education. In the end, some specific reflections as a conclusion that highlight the importance of rebuilding the Latin American University from the recognition of sociospatial realities are presented.

Keywords: Education, Humanities, Socio-spatiality, recognition of the Other

\section{Introducción}

Cuando hablamos de educación estamos directamente señalando la incidencia de ésta en la configuración de los proyectos de sociedad, sin embargo son estos últimos los que a partir de los aparatos institucionales determinan la orientación de las políticas educativas y en gran medida las prácticas pedagógicas, en lo que para Escobar (2007) es la concentración de discursos que fomentan prácticas, generando un impacto sustancial en las relaciones sociales 
entre agentes, no solo relacionados con la educación, sino de la población en general.

Su importancia es más que relevante, al direccionar configuraciones éticas, morales y sociales que en últimas determinan la estructura socioespacial de una comunidad en particular (Machuca, 2013). En lo que para Fleet (2009) en su análisis sobre la legitimación en Weber señala, es la necesidad de la construcción de valores simbólicos generales. En este sentido, la indagación sobre cómo las ciencias humanas han aportado a la configuración de realidades sociales es muy pertinente, ya que permiten dilucidar las relaciones que éstas desde su postura paradigmática han generado en la explicación del mundo social, y que dentro de los marcos de acción política fomentan cohesiones ideológicas.

Las ciencias humanas, en particular las humanidades han tenido un peso vital en la manera de comprender el sujeto, ya sea desde la filosofía, la sociología, antropología o desde los estudios desarrollados por la ciencias sociales, el interés de conocer al sujeto es trasversal a cada una de ellas, sin embargo la orientación y privilegio de ese conocer no siempre pareciera apuntar a los ideales de libertad del sujeto y pleno desarrollo en condiciones de equidad como en algún momento lo invitó la Revolución Francesa (Sánchez, 2005) o las reflexiones comenzando el siglo XX.

Pensar en cómo el conocer social del sujeto ha generado a lo largo de la historia beneficios y grandes tragedias, pensar en cómo ese conocimiento de la realidad social tiene un impacto en la educación y reproducción de estructuras de poder orientadas a objetivos de legitimación de las estructuras económicas, y cómo esta lógica ha difuminado las realidades socioespaciales y de manera particular el reconocimiento del "otro". Es la reflexión a la que invita el texto presentado a continuación.

De tal manera se propone dialogar alrededor del impacto de la relación del conocimiento so-

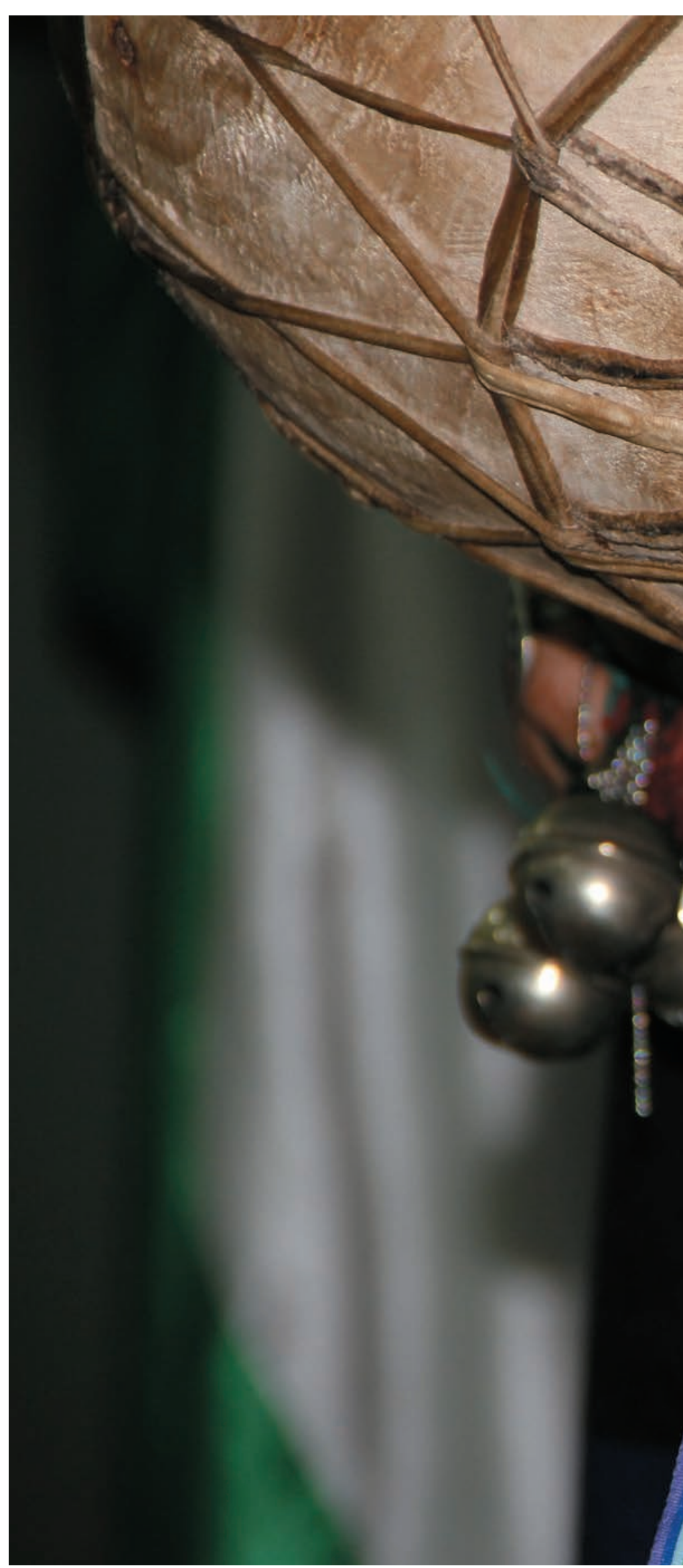




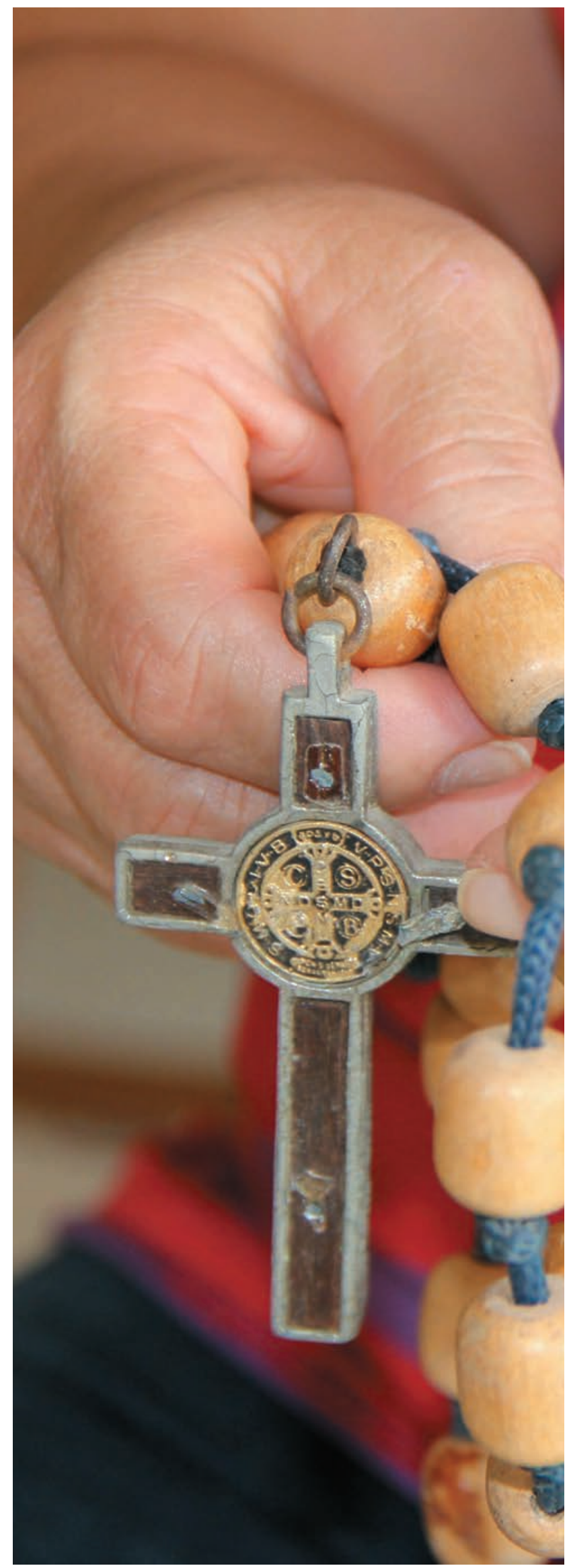

cial y la educación, para lo cual en un primer momento de hará un pequeño reconocimiento histórico de la relación educación y realidades socio-espaciales; esto con el fin de mostrar la ligadura entre ambos y su repercusión en los movimientos sociales, en un segundo momento se pondrá en evidencia el problema de los procesos de colonización en el mundo y particularmente en Latinoamérica. Por último, se señalan los impactos de las políticas educativas, resultado de las lógicas de conocimiento social desarrolladas desde lugares externos, frente a la realidad Latinoamericana y en la comprensión de la educación. Al final se presentan algunas reflexiones puntuales a manera de conclusión.

\section{Desarrollo}

\section{Educación y realidades socio-espaciales}

El desarrollo histórico de las sociedades se ha caracterizado por la utilización de múltiples maneras de transmitir los saberes aprendidos en la experiencia. Esta reproducción de saberes (Terigi, 2007) encuentra su acerbo en la escritura, el lenguaje y en las incontables manifestaciones de la cultura y la tradición. En relación con lo anterior se encuentra que cada una de las representaciones sociales, su historicidad y transmisión están asociadas a la manera como el sujeto asume su territorio, es decir, la manera como genera Topofilias (Tuan, 2007) y construcciones en la trasformación del espacio geográfico como su hogar. Un ejemplo de esto se encuentra en civilizaciones antiguas y americanas.

Al mismo tiempo, en la medida que las necesidades sociales son ampliadas, sus prácticas de reproducción de discursos se complejizan, ya sea por características políticas, económicas o culturales. Las relaciones socio-espaciales establecidas por los sujetos y los mecanismos de reproducción responden la legitimación de acciones políticas por medio de valores simbólicos. La educación resulta ser la estructura más apropiada y por ende la más afectada en térmi- 
nos que muta respecto de los dinamismos sociales propios del desarrollo de las sociedades.

Se puede afirmar entonces, que la educación a lo largo de la historia ha respondido de manera puntual a las dinámicas discursivas del momento histórico en donde está emplazada. Sus postulados han estado ligados a las necesidades sociales imperantes en determinado momento, a la comprensión del espacio geográfico y en general a las relaciones socio-espaciales establecidas por los grupos sociales.

De tal manera, en la antigüedad griega y romana (S. IV a.C.) la educación da inicio a la reflexión con un ideario universal, en la Edad Media (S. XII) su concepción está enmarcada en la fe católica o teológica, en el Renacimiento (S. XVI) aun cuando sigue ligada con el pensamiento cristiano se abre paso dentro del surgimiento del Capitalismo (S. XVIII) y da paso a la educación marcada por el humanismo enfocada en el progreso de los conocimientos para el desarrollo del ciudadano, en la modernidad con un carácter pre-científico caminando hacia su carácter científico gracias a la reflexión filosófica a su alrededor, en el mundo contemporáneo su apertura hacia la crítica y de manera evidente, como respuesta a los sistemas económicos imperantes.

Esta relación que se establece entre la educación y los procesos sociales resulta vital en la configuración de realidades socio-espaciales particulares, si bien la educación se basa en la búsqueda de la transformación para el bienestar de la sociedad, no es ella quien fomenta los cambios, es a partir de sus estructuras políticas la que modifica la filosofía de la educación.

El elemento de legitimación que posee el poder sobre la educación y su impacto en las relaciones contextuales puede significarse de manera puntual atendiendo a los aportes de los Estudios Sociales, al analizar los movimientos. El Marxismo (Boron, 2006) desde la perspectiva del movimiento obrero, deja ver como el elemento de la conciencia resulta determinante en la generación de movimientos y trasformaciones en la sociedad: Si bien el proletario tiene conciencia de su historicidad y de su función en el contexto de lucha, en el ámbito educativo se da una tensión, en tanto que quienes hacen parte del acto educativo no someten sus reflexiones al ámbito dialéctico de tesis, antítesis y síntesis.

Lo anterior se hace evidente, en tanto que la negación de realidades socio-espaciales a lo largo de la historia latinoamericana, fomenta la reproducción de estructuras foráneas y lejanas de asumir procesos de topofilias por el territorio, es decir; no se completa el ejercicio dialectico de formulación de síntesis generadora de conciencia.

Por otro lado, pero continuando con la educación y las realidades socio-espaciales, los aportes de Gustave le Bon a finales del siglo XIX con lo relacionado a la Psicología de Masas, es una evidencia de como la educación genera a partir de su discurso basado en la generación de homogeneidades simbólicas, la pérdida de autonomía por parte del sujeto quien sufre el contagio de las creencias y comportamientos colectivos (Torres, 2009 p. 54).

Es decir, la educación como reproductora de estructuras relacionales con el espacio, suple la necesidad de reconocimiento individual, por un reconocimiento colectivo que en tiempos actuales de globalización responden a la legitimación de acciones políticas a partir de valores simbólicos orientados a la formulación de morales y éticas totalizadoras de la realidad socioespacial excluyendo relaciones particulares entre sujetos: el "otro".

Más dramática es la relación que se establece entre la educación y la teoría del "Agravio Moral" de Barrington Moore citado por Torres (2009), quien se plantea la pregunta orientada a explicar cuál es el elemento que hace que las personas se levanten contra el poder. Cuál 
es entonces, teniendo en cuenta la accidentada y maltrecha historia de Latinoamérica, la situación que generaría un estallido de acciones sociales en pro de la trasformación y reivindicación de las realidades socio-espaciales.

a educación al reproducir los discursos hegemónicos en marcos de éticas totalizadoras, logra niveles de tolerancia y adaptación, fácilmente controlados con mecanismos como las Tecnologías de la Información. La preocupación reside en la articulación de las tres posturas presentadas, que dejan ver la tensa relación entre la educación y las realidades socio-espaciales, en tanto a la reproducción de lógicas de poder y la incompleta generación de agentes de emancipación basados en la conciencia.

Finalmente, son muchas las reflexiones alrededor del sentido de la educación, la enseñanza, la pedagogía y el sinnúmero de disciplinas que se bifurcan de ésta. a preocupación que recae sobre la educación resulta ser el elemento que configura su responsabilidad social. Si bien los planteamientos de los autores señalados frente a las dinámicas sociales plantean lugares de análisis, se presenta una tensión representada en que en su transposición con los procesos educativos y su configuración, no existe el resalte sobre el sujeto latinoamericano y su intrincada realidad socio-espacial. Hecho que pone de manifiesto la necesidad de concretar categorías que abran espacio a los discursos emergidos desde la región y en particular desde sus sujetos, elemento que debe ser evidente en la política pública, tendiente a la reivindicación de posibilidades de cambio.

\section{Latinoamérica: socio-espacialidad negada}

Articulando lo anterior, es importante llamar la atención sobre los procesos de colonización a los que se ve afrontado el contexto latinoamericano, esta colonización que niega las realidades particulares y que por demás genera configuraciones de espacio, cultura y política determinadas a los contextos globales, justifi- cara como se verá en un apartado siguiente las dinámicas educativas.

Para Castro (2000) la modernidad se centraba en la represión de las diferencias, ahora basado en la globalización, son las diferencias las que fortalecen el sistema, tesis que pone de manifiesto los tipos de particularidades. Ya sea desde la ciencia, desde la estructura política o desde las mismas relaciones sociales, las particularidades contextuales son complejas de explicar en momentos de colonialismo intelectual.

El debate sobre el sujeto, su formación y su sentido es para la ciencia algo complejo, conocerlo y quizá manejarlo será un objeto central, razón de esto es ver cómo el Estado desde su estructura, fija el modelo de ciudadano que se debe ser, es decir, desde la oralidad y la escritura se fijan los postulados de estos estándares. Así "Ias ciencias sociales se convirtieron en una pieza fundamental para este proyecto de organización y control de la vida humana" (Castro, 2000 p.90).

Es decir, partimos de una postura en donde lo que importa es homogenizar las características de los sujetos, la ciencias sociales, responderán a las necesidades gubernamentales, explicando la manera como se rigen las categorías sociales, dando paso al estado para intervenir y manejar, enmarcado en la colectividad.

Al mismo tiempo, surge el problema de la creación del "otro", lo que para Castro (2000) supone la necesidad de abordar el proceso de producción material y simbólica, heredado de las sociedades occidentales del siglo XVI, esto resulta efectivo con la legitimación, para el caso latinoamericano, de las constituciones, los manuales de urbanidad y las gramáticas de la lengua. Juntas bifurcadas de los procesos de revolución en Europa, lo cual pone de manifiesto una ligadura colonial de hecho, la escritura es la que genera los niveles de cohesión social en pro de la modernidad. Argumentando la legi- 
timación de instituciones y discursos hegemónicos.

De esta manera y en palabras de Castro (2000, p.91), "la escuela se convierte en un espacio de internamiento donde se forma ese tipo de sujeto que los ideales regulativos de la constitución reclaman", esto como medio para generar una utilidad del sujeto frente a la sociedad. Dentro de lo pedagógico, la escuela cumple con el papel de reproducir la legitimidad de la constitución, del modelo de ciudadano, por ejemplo el Manual de Carreño, elemento de adoctrinamiento y de aseguramiento de progreso. Los manuales dibujan al sujeto CIVITAS, el burgués. Excluyendo al resto. Generando procesos de exclusión social, a partir de la configuración escrita de modelos de civilización.

Lo anterior, asiste a lo que se puede denominar la contradicción de la modernidad "establecer las condiciones para la libertad y el orden implicaba el sometimiento de los instintos, la supresión de la espontaneidad, el control de la diferencias" (Castro, 2000 p.91), contradicción que choca de frente con los ideales propuesto por las revoluciones Europeas, y que en Latinoamérica llegaron como ideales de liberación del sujeto, alimentando deseos de independencia. Para lo cual y como se señaló se requirió el control.

Esta concepción de libertad, basada en el control, en la supresión de los instintos y por demás en las prácticas de poder sobre el "otro", son la realidad de una región que no tiene una epistemología de su propia configuración socio-espacial. Su característica más aberrante es la homogenización de realidades, lo que supone la construcción de imaginarios de poder sobre lo bueno y lo malo. Posicionando la denominada "violencia epistémica" (Castro, 2000).

En concordancia hay que comprender que los estados nacionales, no surgen por autonomía, sino por relación directa a los procesos colonialistas en ultramar. Históricamente se ha

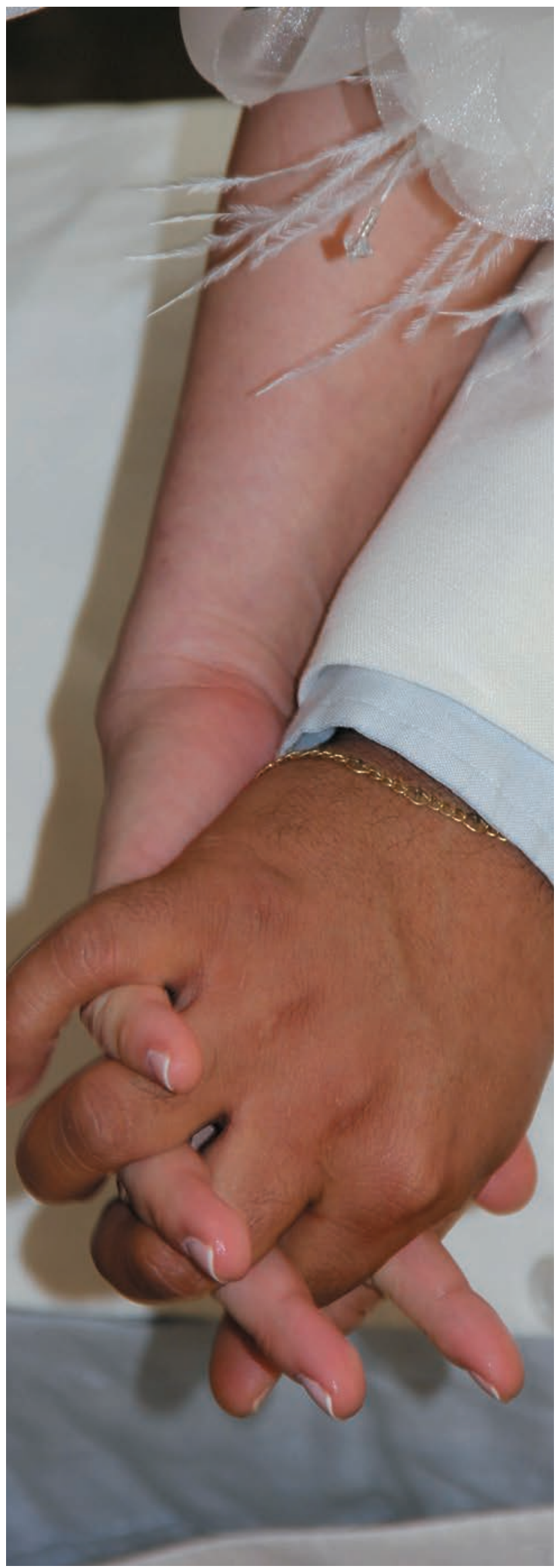


formulado, desde la ciencias sociales, la creencia de la necesidad de la colonización, y la autonomía del desarrollo de Europa, es decir se niegan las relaciones socio-espaciales que se dan en estos procesos "lo cual significa (...) que el colonialismo no significó destrucción y expoliación sino, ante todo, el comienzo del tortuoso pero inevitable camino hacia el desarrollo y la modernización" (Castro, 2000 p.92).

Razón por la cual no se puede negar el influjo que tuvo los procesos de colonización, elemento que no tiene en cuenta la reproducción que hacen las ciencias sociales. O lo que se puede denominar el poder disciplinario, que según Foucault (Castro, 2014, p.92) es una característica de las sociedades modernas, es decir, que el estado moderno está relacionado con el sistema internacional de poder. Esto comprendido desde una lógica actual de globalización.

Se plantea así que la post-modernidad, busca el desarrollo aparentemente pleno del sujeto, es decir, ya no existe la necesidad de homogenizar, en ella siempre hay posibilidad. "la producción de bienes simbólicos y por la seducción irresistible que estos ejercen sobre el imaginario" general el poder libidinal (Castro, 2000 p.94). Las ciencias humanas, contribuyen a la diferenciación, y a la homogenización institucional.

El lenguaje, entonces aparece como elemento de unión y diferenciación, o lo que para Lyotard citado por Castro (2000 p.94) "En la condición posmoderna, son los jugadores mismos quienes construyen las reglas de juego que desean jugar. No existen reglas definidas de antemano". Algo que utilizara muy bien el complejo económico industrial para justificar una educación flexible y competitiva.

La argumentación expresada, evidencia como la realidad socio-espacial de Latinoamérica no está en marcos de conciencia histórica subjetiva, sino que al contrario está fundada en parámetros foráneos que no atienden a las

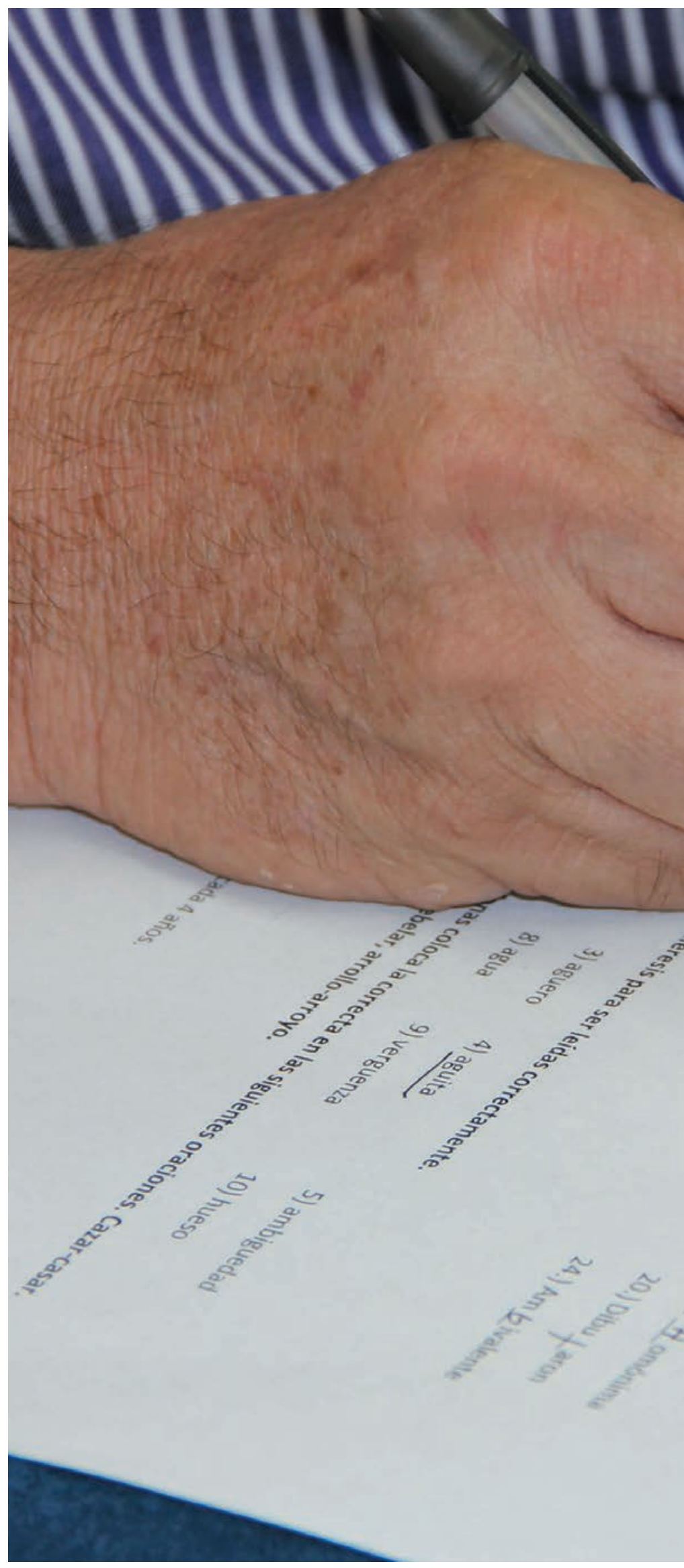


para entrar desde la educación a esta lógica de mercado. La educación es víctima de los intereses acelerados de los gobiernos de entrar a la globalización, sin tener en cuenta las capacidades no solo industriales, sino culturales tan distintas y ricas que existen en esta región.

La concepción de enmarcar al sujeto dentro de la flexibilidad para la producción de bienes y servicios, pone de manifiesto el paradigma de competencia en la educación hoy, en una política globalizadora de la educación donde ésta debe aportar de manera directa a los modelos de producción, un claro ejemplo es el proyecto Tuning Latinoamérica, que se conecta directamente con el Tuning Europeo.

La educación asume como un insumo para la producción de capital, hecho que le resta autonomía a cada uno de sus componentes (Escuela, Universidad, entre otros) elemento que señala la ligadura tan fuerte que existe con la cultura occidental. Se pueden señalar tres momentos claves en esta relación para Charlot (1987) citado por Acosta (2007): 1. Integración moral, lingüística y política en su nacimiento, 2. Imperativo industrial que determina casi que su configuración actual y 3 . Sociedad de mercado que detalla la escuela moderna.

Los procesos históricos tienen un paso sobre las estructuras curriculares así se pasa de una postura del saber a una postura del saber utilitario (comprendiendo que el saber debe servir para acumular) en lo que para Laval (2004) citando a Spencer representa la exclusión de saberes, en donde se da relevancia a componentes que en la práctica de la escuela no tienen mayor relevancia, fomentando el descuido de otros que resaltan por su importancia en la vida.

La escuela (y aquí se entiende por escuela el espacio de socialización, reproducción y critica de los saberes, lo cual incluye a toda estructura educativa), pasa al servicio de lo económico, efecto de la necesidad de la década de los se- sentas y setentas, que supuso una crisis energética poniendo en "riesgo" el procesos social, esto impulsa la restructuración de las políticas educativas, convirtiendo la educación en "en una verdadera industria de masas, no podrá ser descrita sistemáticamente sino con ayuda de categorías económicas" (Laval, 2004 p.45).

Lo expuesto pone tres ejes sobre la mesa, respecto de la función de la educación, el primero la formación de mano de obra, el segundo la transformación cultural y el tercero la formación de ciudadanos responsables. La escuela aquí, deja de ser la única que posee el conocimiento, es la realidad global la que lo posee, razón que junto con las políticas desarrolladas, justifica su inserción en las lógicas del mercado. Ejemplificado esto con la estandarización, descentralización y la mutación de la gestión empresarial, las reformas así apuntan a ver "la empresa escolar" (Laval, 2004 p.48).

El sujeto entonces entra en la lógica de la adaptación, si la educación está en un lógica económica, debe producir, por ello no solo ésta debe adaptarse al mercado y sus necesidades, el sujeto de igual manera, "el ideal de la escuela es en adelante el trabajador flexible" (Laval, 2004 p.53). Poniendo de manifiesto la generación de nuevos valores sociales, como la eficacia, la movilidad y el éxito personal.

Estos nuevos valores, ponen al sujeto como un elemento que dentro de esta flexibilidad se caracteriza por no ser líder, sino competente y adaptable a diferentes situaciones, esta flexibilidad supone que la escuela asuma los discursos de movilidad curricular, preparándolo para la incertidumbre, dotándolo de competencias organizativas, comunicativas de adaptabilidad, trabajo en equipo y resolución de problemas en contextos complejos, es decir aprender a aprender.

Así, la educación para toda la vida fundamentada en la concepción de que el conocimiento es global y está en constante cambio, donde la 
empresa también enseña. Pone de manifiesto que el "titulo" es un elemento estático que pierde validez y diferenciación social, esto porque hoy en la sociedad de la información el conocimiento es perecedero "el titulo escolar y universitario, en una época en la que se declara que el saber es un producto perecedero, y que las competencias mismas son objeto de una destrucción creadora permanente, tiende a perder su fuerza simbólica" (Laval, 2004 p. 52).

El saber es entonces útil para la eficacia laboral, el modelo económico imperante necesita de capital humano formado para tal, así el formar a lo largo de la vida resulta fundamental, o como lo señala el ERT (1997) citado por Laval (2004, p.85) "la población... debe empeñarse en un proceso de aprendizaje a lo largo de la vida. La Integración creciente del conocimiento en el entorno industrial transforma a los trabajadores en trabajadores cognitivos".

De esta manera, los saberes deben enfocarse a lo técnico, es decir a lo directamente comprobable y beneficioso en la producción de bienes y servicios, esta educación debe entonces articularse a las estructuras de producción de la empresa. Se habla de capital humano entonces, razón que explica la manera de comprender la educación, como un elemento de la producción, por ende debe generar algún beneficio.

La investigación, uno de los principios de la educación (para el caso puntual de la Universidad), cae de igual manera en esta lógica, se toma entonces como una inversión, para que sus resultados alimenten las lógicas del mercado, esto se hace evidente en la subordinación de saberes a la económica, con la multiplicación de inversiones en I+D y patentes (Laval, 2004). Un claro ejemplo es el modelo Norteamericano, que resulta de la competencia misma contra el acelerado crecimiento de Japón, quien luego del Crimen de la Bombas Atómicas al finalizar la Segunda Guerra Mundial, se recuperó de manera asombrosa (la educación como

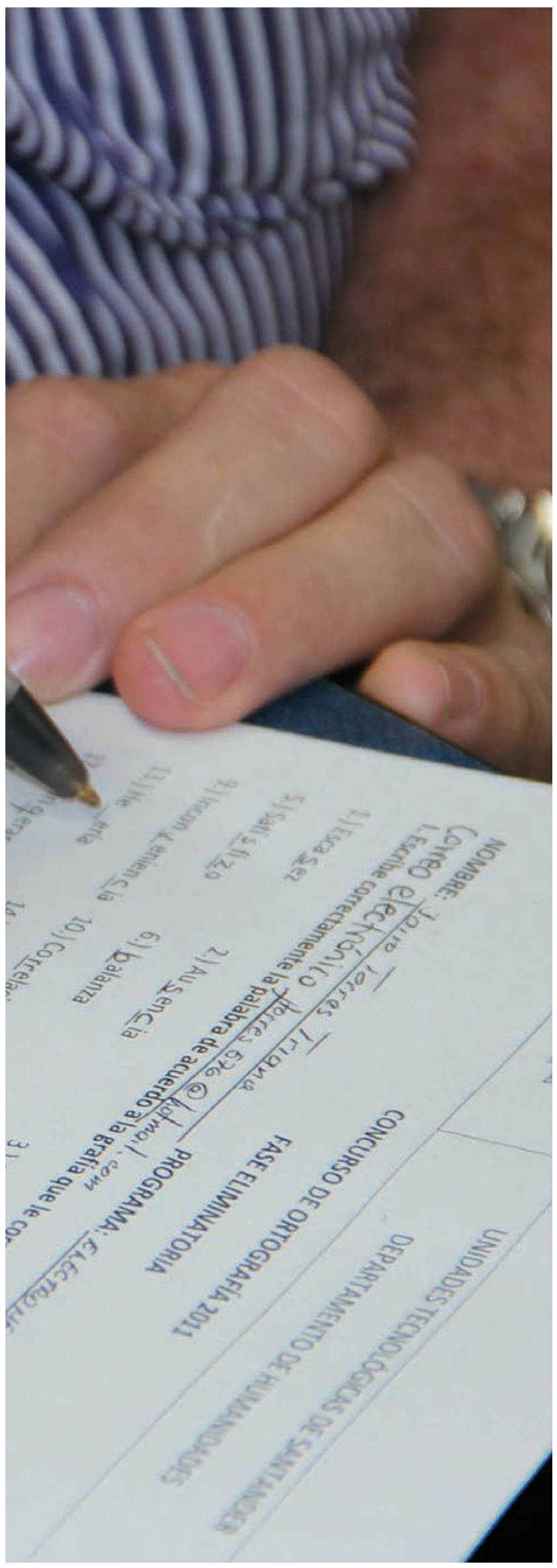




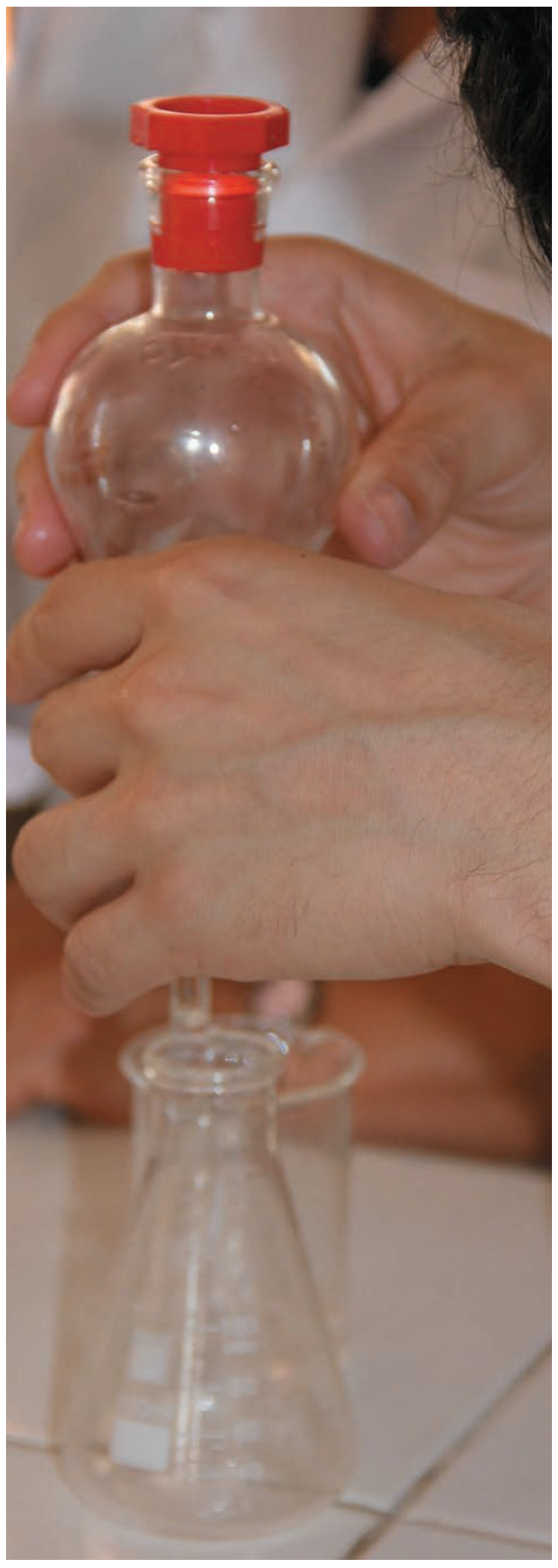

necesidad de potencializar sus discursos imperialistas).

Razón por la que, el modelo de inversión de la empresa privada a los centros de educación para investigación, se convirtió quizá en una afrenta a la filosofía de la universidad, ya que se convierten en fábricas del saber empresarial. Esto tiene una consecuencia contundente, su autonomía se ve cuestionada, la empresa toma decisiones sobre sus planes de estudio, enfoques de las investigaciones y hasta en lo pedagógico.

Continuando con lo anterior, es evidente que pensar la educación desde categorías económicas afecta en el conjunto de su configuración, sus acciones se ven restringidas a la creación de agentes y conocimientos para la generación de bienes y servicios o en lo que para Andre Tosel es "la escuela desemancipadora" (Laval, 2004). Algo evidente en los contextos latinoamericanos, donde si bien esta inserción de modelos y tendencias buscan la transformación social enmarcada en la igualdad, la realidad dista mucho de este discurso, que por demás es una negación a las condiciones culturales de una región con grandes complejidades que no ha logrado posicionarse dignamente en el mundo.

Las prácticas docentes (Londoño, 2010), se ven afectadas ya que estas deben por ende responder a las necesidades de la empresa, a las necesidades del mercado. Más no a las necesidades de lo social. Apoyado todo esto en una retórica fundamentada en el capitalismo flexible que arguye a su carácter liberador. Es así como no basta con la política económica educativa, es necesario generar la infraestructura necesaria para que ella funcione.

De manera general y según lo que Laval (2004) plantea en su texto, los nuevos modelos económicos, tienen un peso determinante en todas las realidades humanas. La educación es una de ellas debido a que los discursos 
se difunden bajo una lógica de justificación y de igualdad entre agentes, que confluyen hacia los procesos de producción. La escuela se mercantiliza al convertirse en una herramienta más del sistema económico, preparando en las competencias que se requieren en la producción y por demás en la conciencia de agentes consumidores.

El docente entonces, se ve en esta compleja red empresarial, que pone lo pedagógico como elemento para asegurar procesos de enseñanza propicios a las necesidades ya mencionadas (Machuca, 2013). Todo el sistema educativo cae en una lógica productiva y competitiva que asalta de manera evidente su compromiso de transformador de la sociedad, al convertirla en la generadora de capital humano para las labores industriales, que se sitúan lejos de las realidades socio-espaciales.

Resultado de lo anterior es la configuración misma de la universidad latinoamericana, que resulta de la mezcla de modelos foráneos, desarticulados de las realidades socio-espaciales y que propenden por la reproducción de lógicas economicistas que se pueden evidenciar en los enclaves discursivos de la universidad en Latinoamérica (Borrero, 2008) y su vinculo excesivo con modelos como el alemán, francés y norteamericano.

\section{Conclusiones}

Hablar de Latinoamérica sin lugar a dudas requiere hablar del "desarrollo" histórico de la humanidad, si bien los contextos cuentan historias, son estos mismos contextos junto con sus cargas socio-espaciales los que expanden discursos totalizadores aun en contextos opuestos. Es evidente que Latinoamérica ha sido una reproductora de discursos, que están fuera de sus realidades contextuales, que no son precisamente las de la ilustración, la revolución industrial o la revolución burguesa. Existía una sociedad compuesta por realidades diferentes a las vividas en Europa antes de la conquista.

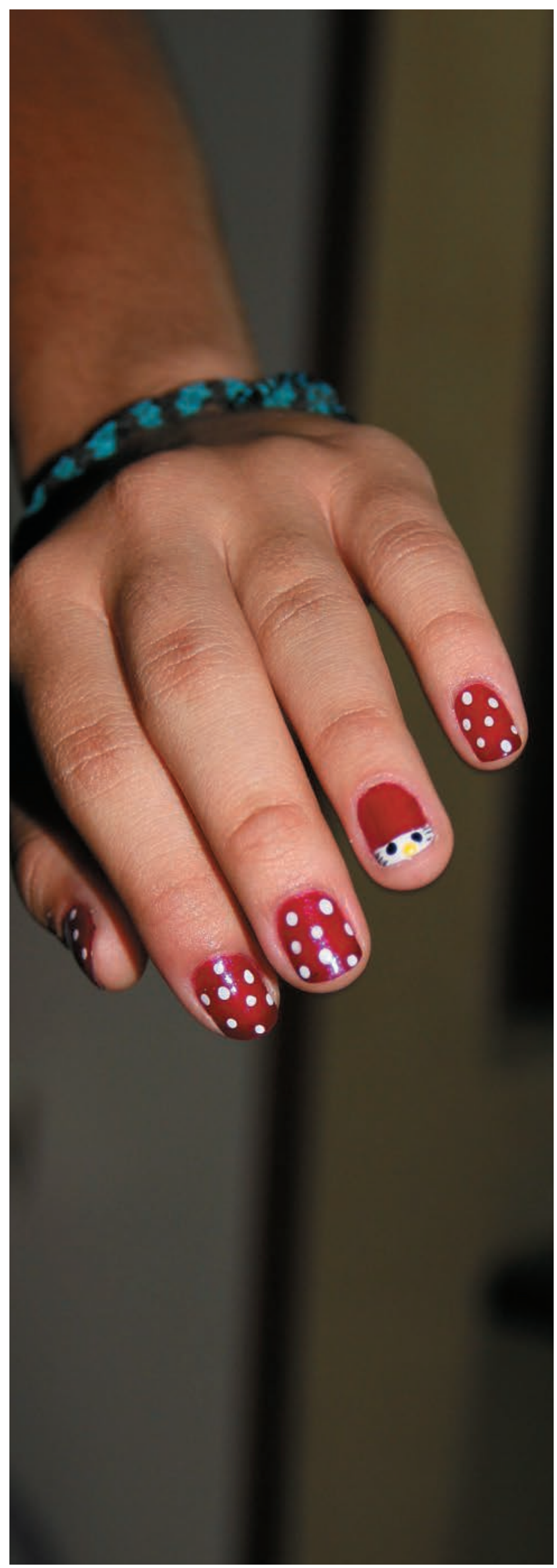


De esta manera, cabe reflexionar sobre el papel de la educación como reproductora de saberes descontextualizados, poco críticos y por demás con una decidida por la transformación real de la sociedad en pro de la ruptura de tradiciones históricas poco benéficas para una sociedad afectada de manera contundente por estos discursos. Es necesaria la puesta en juego de la conciencia histórica latinoamericana, de un rescate por el Ser desde los contextos y realidades socio-espaciales particulares de esta región, elementos que posibilitan la transformación social, y que es compromiso social de la educación, por ende del docente.

La necesidad de comprender las representaciones sociales como elementos que posibilitan en la territorialidad acciones, implica resaltar el papel que la educación tiene dentro del entramado social. El reconocimiento de las realidades socio-espaciales dentro de la educación superior latinoamericana requiere dar un paso al frente y empoderarse de los procesos espacio-temporales que subyacen a las lógicas hegemónicas orientadas a lo económico.

He llegado a este punto para mostrar como el ser docente de humanidades, significa más que una simple transmisión de conocimientos de historia o geografía, es problematizar frente a la realidad misma de los estudiantes que rodean la práctica pedagógica (Machuca, 2013). Este saber pedagógico se pone en juego todos los días y se conjuga en la práctica pedagógica, que se asume desde el reto presentado: asumir la conciencia histórica como elemento para el rescatar el Ser.

\section{Referencias bibliográficas}

Acosta, W. (2007) El nuevo orden educativo global y la mercantilización de la escuela pública en Bogotá 1990 2004. Bogotá: Universidad Pedagógica Nacional.

Boron, A. (2006) La teoría Marxista Hoy. Problemas y Perspectivas. Colección Campus Virtual. CLASCO. Buenos Aires, Argentina. ISBN: 987-1183-52-6
Borrero, A. (2008) La Universidad: estudios sobre sus orígenes, dinámicas y tendencias. Bogotá: Tomo III, Compañía de Jesús y Pontificia Universidad.

Castro-Gómez, S (2000). Ciencias sociales, violencia epistémica y el problema de la "invención del otro". En libro: La colonialidad del saber: eurocentrismo y ciencias sociales. Perspectivas Latinoamericanas. Edgardo Lander (comp.) CLACSO, Consejo Latinoamericano de Ciencias Sociales, Buenos Aires, Argentina., p 88-98. Disponible en: http://biblioteca.clacso.edu.ar/gsdl/collect/ clacso/index/assoc/D1334.dir/lander.pdf

Castro, E. (2014) Introducción A Foucault. Buenos Aires: Siglo Veintiuno Editores.

Escobar, A. (2007) La invención del tercer mundo. Caracas: Fundación Editorial el Perro y la Rana.

Fleet, N. (2009) Razón y dominación. La legitimidad en Weber como orientación simbólica de la acción política. Revista Austral de Ciencias Sociales, núm. 16, 2009, pp. 21-36,

Laval. C. (2004) La escuela no es una empresa. Barcelona: Paidós.

Londoño, G. (2010). Docentes Ámbito Universitario. El saber pedagógico componente fundamental en la docencia universitaria. Bogotá: Ed. Unisalle.

Machuca, G. (2013) Estrategias de mediación para la comprensión de la escuela como un sistema socio-espacial: aporte para la formación docente. Bogotá: Ediciones Universidad Libre.

Sánchez, R. (2005) El sentido de la Revolución Francesa y sus utopías. En Praxis Filosófica: Nueva serie, No. 20, Ene.Jun. 2005: 87-112. ISSN: 0120-4688. Bogotá, Colombia

Terigi, F. (2007). Exploración de una idea. En torno a los saberes sobre lo escolar. En Frigerio, G., Diker, G. y Baquero, R. (comps.), Lo escolar y sus formas, Buenos Aires, Del Estante Editorial.

Torres, A. (2009) Acción colectiva y subjetividad. Un balance desde los estudios sociales. Revista FOLIOS, núm. 30, 2009, pp. 51-74, Universidad Pedagógica Nacional.

Tuan, Y. (2007) Topofilias: Un Estudio De Las Percepciones, Actitudes Y Valores Sobre El Entorno. Traducción del inglés de Flor Duran de Zapata, $1^{\text {a }}$ ed., $1^{\text {a }}$ imp. Editorial Melusina. España.

Vega, R. (2011). Un mundo incierto, un mundo para aprender y enseñar: Imperialismo, geopolítica y retórica democrática. Bogotá: Universidad Pedagógica Nacional. 